\title{
GESTÃO SUSTENTÁVEL: OS PARADIGMAS DO NOVO CONTEXTO EMPRESARIAL
}

\author{
SUSTAINABLE MANAGEMENT: THE PARADIGMS OF THE NEW BUSINESS \\ CONTEXT
}

\author{
Helena Karoline da Paz Silva ${ }^{1}$ \\ Shynaide Mafra Holanda Maia ${ }^{2}$ \\ José Luiz Alves ${ }^{3}$
}

RESUMO: A gestão corporativa sustentável tem se tornado a cada dia um grande diferencial nas empresas. A partir de uma revisão de literatura o presente artigo conceitua três modelos de mensuração de sustentabilidade empresarial visando descortinar os paradigmas enfrentados para análise da sustentabilidade das organizações. O tema é de suma relevância, pois a sustentabilidade é uma necessidade e inexiste um padrão de indicadores para medi-la, fazendo necessária a ampliação dos debates relacionados ao tema os quais podem ser utilizados como base para a definição de estudos mais aprofundados

Palavras-chaves: Gestão corporativa. Sustentabilidade. Indicadores.

ABSTRACT: Sustainable corporate management has become every day a great differential in companies. Based on a literature review, this article defines three models for measuring corporate sustainability in order to unveil the paradigms faced for analyzing the sustainability of organizations. The theme is extremely relevant, as sustainability is a necessity and there is no standard of indicators to measure it, making it necessary to expand the debates related to the theme, which can be used as a basis for the definition of more in-depth studies.

Keywords: Corporate management. Sustainability. Indicators.

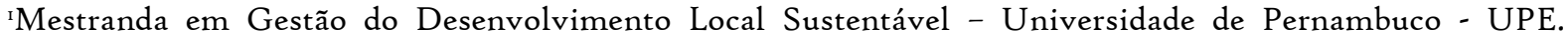
Especialista em Gestão de Negócios (UPE). Administradora (UPE). Endereço eletrônico: Email: helenaksilva@gmail.comhttp://lattes.cnpq.br/7553661473138599https://orcid.org/oooo-ooo2-7480-5317 ${ }^{2}$ Mestranda em Gestão do Desenvolvimento Local Sustentável - Universidade de Pernambuco - UPE. Especialista em Direito Previdenciário. Professora de Direito Previdenciário da ESMATRA TRT6. Advogada. Email: shynaide@smafra.com.br. http://lattes.cnpq.br/1651995864356392https://orcid.org/oooo0003-1965-720

3 Prof. Adjunto Universidade de Pernambuco - Universidade de Pernambuco - UPE. Doutor em Geografia Universidade Federal de Pernambuco - UFPE. Mestre em Geografia - UFPE. Bacharel em Ciências Econômicas - $\quad$ UFPE. Endereço eletrônico: luiz.alves@upe.br. http://lattes.cnpq.br/7078869287819893https://orcid.org/oooo-ooo3-2049-2084.
} 


\section{INTRODUÇÃO}

A sustentabilidade apresenta-se no mercado atual como um diferencial competitivo que pressiona as organizações a seguir um novo caminho, reestruturando suas atividades organizacionais, operacionais, estratégicas e tecnológicas. A dedicação para mostrar-se pioneira e se destacar dentre inovações sustentáveis, tem como objetivo agregar valor à organização e criar o conceito de valor sustentável, se não existente anteriormente, sob o preceito das três dimensões: economia, sociedade e meio ambiente (AKTAS; KAYALIDERE; KARGIN, 2013).

Por ser algo tão novo e emergente, os conceitos de desenvolvimento sustentável na literatura apresentam diversos tópicos, que a depender do foco da organização podem ganhar mais destaque no seu processo de criação de valor. Por exemplo, para Luke (2005) o mercado usa as palavras e termos ligados à sustentabilidade de maneira sofisticada, astuta e confusa buscando produzir efeitos de poder naqueles que a recebem. Enquanto Finkbeiner, Schau, Lehmann e Traverso (2010) consideram a sustentabilidade como impulsionador de políticas públicas e boas estratégias corporativas, agindo por meio de seus atores. No mesmo sentido, Van Marrewijk and Werre (2003) afirma que a sustentabilidade só é alcançada quando se muda a cultura. Janeiro e Patel (2015) evoluíram o conceito de processos de sustentabilidade com o uso de tecnologias nos ambientes organizacionais levando em consideração os indicadores racionais, econômicos e sociais.

Em seu estudo, Stival e Sottoriva (2015) demonstram que o governo brasileiro instituiu normativas e instruções para a adoção de critérios de sustentabilidade, considerando todos os processos desde a extração/fabricação até a comercialização do produto. Neste ambiente, a empresa $B_{3}$ - que é uma companhia de infraestrutura de mercado financeiro - criou um índice para medir a sustentabilidade de empresas de capital aberto no Brasil, denominando-o Índice de Sustentabilidade Empresarial (ISE).

Nesta lógica, observou-se a presença de diversos modelos de mensuração de sustentabilidade e, conforme apresentado por Costa et al. (2017), estes modelos são separados de forma que possuem análise numérica ou teórica. Como o intuito deste trabalho é analisar os paradigmas encontrados dentro do contexto empresarial atual para a gestão sustentável, seguiu-se o foco teórico das análises buscando ter uma análise atualizada dos paradigmas que as organizações enfrentam para serem sustentáveis. Esta 
pesquisa mostra-se relevante, pois o mercado encontra-se em evolução e a sustentabilidade é um diferencial competitivo. Dessa forma, busca-se dar um panorama das teorias mais atuais para suporte as implementações de índices sustentáveis.

Além da introdução, este trabalho conta com mais quatro sessões. $\mathrm{Na}$ primeira, temos a base teórica abrangendo os conceitos de sustentabilidade e gestão empresarial sustentável; na segunda, a explanação da metodologia aplicada na execução da pesquisa; na terceira parte, a explanação dos métodos de medição teóricos de sustentabilidade; e por fim, as conclusões.

\section{BASE TEÓRICA}

\section{O conceito de sustentabilidade e o "Triple Bottom Line"}

O conceito de desenvolvimento é amplamente utilizado por diversos meios e em diversos contextos e passou por diversas mudanças ao longo do tempo e vários adjetivos foram incorporados a ele, para melhor qualificá-lo, como "social”, "humano", “econômico”, “sustentável”, “local”, entre outros (KRONEMBERG, 2019).

O desenvolvimento sustentável é aquele que atende às necessidades do presente sem comprometer as necessidades da geração futura. Este desenvolvimento supõe uma mudança progressiva da economia e da sociedade. Satisfazer as necessidades e aspirações humanas é o principal objetivo do desenvolvimento, entretanto, balancear o crescimento econômico com as necessidades a serem atendidas, buscando igualar as diferenças préexistentes no ambiente (BRUNDTLAND, 1989).

A sustentabilidade, por definição, é uma meta de política composta e, portanto, ambiciosa. Compreende critérios ambientais, econômicos e sociais de igual importância nenhuma degradação ambiental nem violação da dignidade humana pela pobreza ou outras ameaças, nem públicas ou privadas. (AZAPAGIC, A.; PERDAN, S., 2000).

Elkington (1994) desenvolveu o conceito de sustentabilidade mais reconhecido e adotado, o "triple bottom line". Esse conceito distingue e propõe uma abordagem equilibrada para aspectos econômicos, ambientais e sociais do desempenho empresarial (Gimenez et al., 2012). Dias (2019) esclarece que o Triple Bottom Line também é conhecido como os ${ }_{3} \mathrm{Ps}$ (People, Planet and Profit) e no Brasil é nomeado como tripé da sustentabilidade. O conceito de Triple Bottom Line está intimamente relacionado com a 
invasão da Responsabilidade Social no âmbito corporativo que aumentou a exigência pela incorporação de novos indicadores a fim de quantificar o impacto das empresas sobre os stakeholders externos.

Segundo Nogueira e Faria (2012), a dimensão econômica instrui para que as companhias proporcionem melhores condições de trabalho, sejam economicamente viáveis e respeitem as diversidades culturais. O que já interliga com a dimensão social, que traz para a organização seu envolvimento nas atividades socioculturais da comunidade. $\mathrm{E}$ por fim, na dimensão ambiental, estabelece o respeito ao meio ambiente e uma gestão ecológica dos recursos.

\section{Gestão ambiental empresarial}

\begin{tabular}{|c|l|}
\hline \multirow{2}{*}{ Qual a origem da divulgação? } & Obrigação legal \\
\cline { 2 - 3 } & Ato voluntário \\
\hline \multirow{2}{*}{ Para quem divulgar? } & \\
\hline \multirow{2}{*}{ O que divulgar? } & Partes interessadas específicas \\
\cline { 2 - 3 } & Público em geral \\
\hline \multirow{2}{*}{ Como divulgar? } & Exclusivamente questões ambientais \\
\hline & Questões ambientais, sociais, econômicas etc. \\
\hline & Modelo próprio \\
\hline & \\
\cline { 2 - 3 } & Modelo padronizado por outras organizações \\
\hline
\end{tabular}

Quadro or: Barbieri (2016)

A solução dos problemas ambientais, ou sua minimização, exige uma nova atitude dos empresários e administradores, que devem passar a considerar o meio 
ambiente em suas decisões e adotar concepções administrativas e tecnológicas que contribuam para ampliar a capacidade de suporte do planeta (BARBIERI, 2016).

Segundo Tachizawa (2019) um dos maiores desafios que o mundo enfrenta neste novo milênio é fazer com que as forças de mercados protejam e melhorem a qualidade do ambiente, com ajuda de padrões baseados no desempenho e uso criterioso de instrumentos econômicos, num quadro harmonioso de regulamentação.

$\mathrm{Na}$ visão de Dias (2019), existem motivos diversos que podem incentivar uma empresa a adotar metodologias de gestão ambiental, afora os interesses econômicos, como a necessidade de redução de custos, melhoria na imagem do produto e da empresa, necessidade de inovação, aumento da responsabilidade social, demanda de mercado, certificações ambientais, além da concorrência.

O renomado Tachizawa (2019) acrescenta que uma gestão ambiental e de responsabilidade social consiste no exame e revisão das operações de uma organização da perspectiva do novo paradigma.

Barbieri (2016) esclarece que comunicações e relatos ambientais, veiculados por qualquer meio, objetivam divulgar os aspectos ambientais da organização, seus impactos e o que ela faz e pretende fazer em relação a eles. Diz ainda que existem diversos modelos de relatórios de sustentabilidade, destacando o GRI (Global Reporting Initiative) e também

sobrelevando que cada organização se amolda a uma demanda específica, conforme quadro esquemático abaixo.

Considerando a necessidade de medir, monitorar e avaliar os avanços das empresas em relação à sustentabilidade, vários modelos e indicadores foram criados a fim de permitir atualmente a mensuração da sustentabilidade empresarial, embora, nenhum desses métodos são suficientes para serem utilizados por empresas de diferentes setores (SZÉKELY E KNIRSCH, 2005).

\section{METODOLOGIA}

O presente trabalho se caracteriza como de natureza qualitativa e exploratória, pois são realizadas investigações de pesquisa, cujo objetivo é a formulação de questões ou de um problema. A metodologia qualitativa ajuda na compreensão do processo estudado e possui como instrumento a observação sistemática (MARCONI, 202I). 
Os dados coletados neste estudo, compreendem uma análise de quatro modelos de mensuração de sustentabilidade empresarial com o objetivo de enxergar os paradigmas enfrentados para analisar a sustentabilidade das organizações. Para Costa et al. (2017), ao longo dos anos foi gerada a necessidade de mensurar além da produtividade, a confiabilidade, eficiência e sustentabilidade nas empresas.

A inexistência de um conjunto de indicadores aceitos de forma ampla, dificulta a mensuração da sustentabilidade nas organizações (SEARCY; KARAPETROVIC; MCCARTNEY, 2005; DELAI; TAKAHASHI, 2008). A medição da sustentabilidade ainda não consegue ser realizada de maneira a atingir de forma igualitária e precisa suas três dimensões (ANDRADE; CÂMARA, 2012). Dessa forma, é necessário a aplicabilidade de metodologias conforme os segmentos e atuações da organização no mercado (SZÉKELY; KNIRSCH, 2005).

\section{RESULTADOS E DISCUSSÕES}

\subsection{Modelos de Mensuração de Sustentabilidade Empresarial}

3.I.I. Modelo de Spangenberg e Bonniot (1998)

Spangenberg e Bonniot consideram que a sustentabilidade, por definição, é uma meta de política composta e ambiciosa, compreendendo critérios ambientais, econômicos e sociais de igual importância. A dimensão física da sustentabilidade refere-se a deixar intacto - por um período infinito de tempo - a estabilidade dos processos evolutivos internos da ecosfera, uma dinâmica e auto-organizada estrutura. Um sistema econômico é ambientalmente sustentável apenas enquanto a quantidade de recursos utilizados para gerar bem-estar é permanentemente restrita a um tamanho e uma qualidade que não super explore as fontes ou sobrecarregue os sumidouros fornecidos pela ecosfera.

Em função das dificuldades, um conceito normativo coerente de desenvolvimento sustentável, incluindo um a análise de custo-benefício das estratégias políticas é uma contradição em termos, mas o que se pode tentar é fornecer a todos os atores dois novos tipos de ferramentas que ajudam a direcionar a tomada de decisões para sustentabilidade: por um lado, uma visão de uma sociedade sustentável, útil como uma bússola, não um mapa de rotas e, por outro lado, indicadores que ajudam a medir progresso, distância do 
alvo e falhas de planos ou de suas implementações. Assim, os autores elaboraram o modelo abaixo:

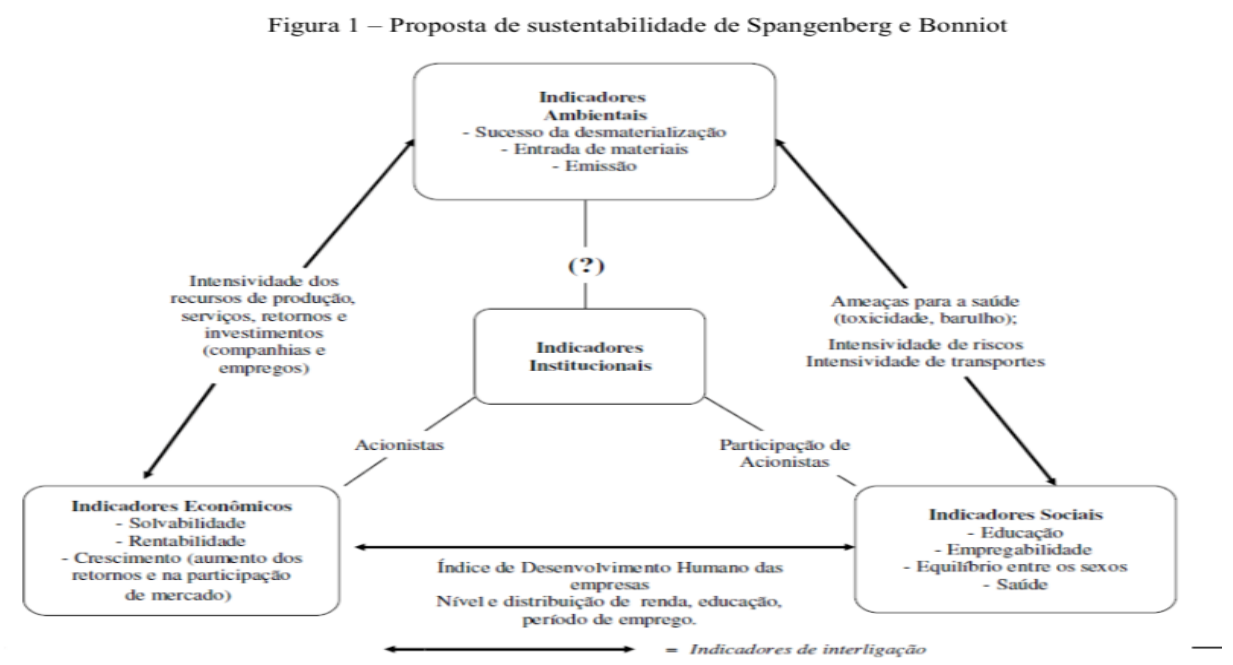

Figura or: Spangenberg e Bonniot (1998)

\subsubsection{Modelo de Azapagic e Perdan (2000)}

Os indicadores padronizados pelos autores permitiriam a identificação de opções mais sustentáveis por meio de: comparação de produtos semelhantes feitos por diferentes empresas; comparação de diferentes processos produzindo os mesmos produtos; benchmarking de unidades dentro das corporações; classificação de uma empresa em relação a outras empresas no (sub) setor; e avaliação do progresso em direção ao desenvolvimento sustentável de um (sub) setor.

Além de poder comparar os níveis de sustentabilidade de diferentes sistemas, os indicadores propostos também podem medir o progresso relativo em direção (ou afastamento) do desenvolvimento sustentável. Assim, os indicadores relativos que medem o progresso regularmente são mais úteis, pois permitem que empresas e consumidores rastreiem melhorias (ou desvios da sustentabilidade curso) de ano para ano e identifiquem opções e práticas mais sustentáveis.

Existem duas etapas na padronização dos indicadores. Em primeiro lugar, os indicadores que fundamentam os princípios do desenvolvimento sustentável devem ser identificados. Eles incluem indicadores genéricos para todos os indicadores da indústria e setores específicos. O setor específico dos indicadores irá variar de setor para setor, e eles 
são melhores identificados caso a caso. A segunda etapa da padronização está relacionada a identificar as métricas apropriadas que permitiriam o rastreamento de desempenho e a comparação de diferentes opções.

Os autores apresentaram métricas diferentes em relação ao tipo e objetivo da análise e sugerem em seu modelo três possibilidades de análises: (I) orientada ao produto; (2) orientada ao processo e (3) orientada à pesquisa.

\subsubsection{Modelo de Dyllick e Hockerts (2002)}

A nível empresarial, Dyllick e Hockerts (2002) expõem que a sustentabilidade é frequentemente equiparada à eco-eficiência. E a partir dos três tipos de capital econômico, natural e social - desenvolveram seis critérios de satisfação a serem aplicados pelos gestores que buscam a sustentabilidade empresarial: eco-eficiência, socio-eficiência, eco-eficácia, socio-eficácia, suficiência e equidade ecológica.

A figura 02 nos permite ter uma visão geral do relacionamento entre os seis critérios de satisfação trazidos por Dyllick e Hockerts (2002). Os capitais sociais, econômicos e natural, geram em cada esfera de relacionamento duas variáveis que necessitam estar em equilíbrio para assim alcançar a sustentabilidade dentro da organização. O contraponto é que a execução desses critérios diverge nos resultados sustentáveis, exigindo ainda mais dos gestores envolvidos no processo clareza e comprometimento com os objetivos, como melhor poderemos ver abaixo:

Figura 02: Visualização dos seis critérios de sustentabilidade empresarial. Adaptado de

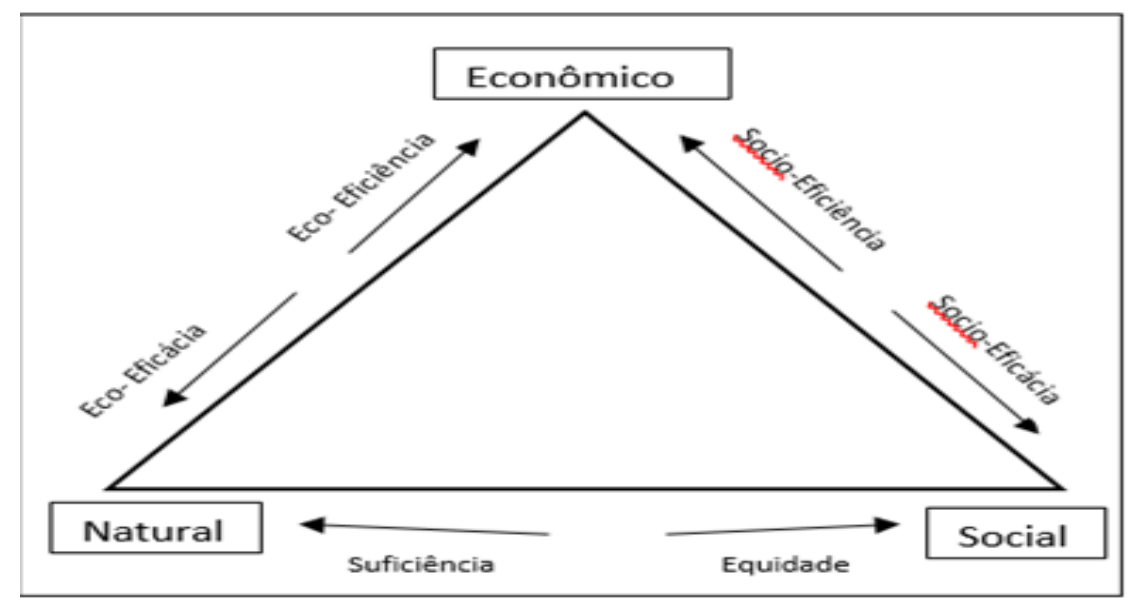

Dyllick e Hockerts (2002). 
I) Eco-Eficiência: Caracteriza-se pelo uso eficiente dos capitais naturais da organização, ou seja, quando esta consegue atingir sua produção de bens e serviços com preços competitivos satisfatórios para a necessidade humana, reduzindo os impactos ecológicos e o uso intensivo dos recursos naturais ao longo do ciclo de produção, respeitando a capacidade do planeta.

2) Sócio-Eficiência: Caracteriza-se pelo impacto social causado pela empresa no ambiente em que se encontra. Podem ser classificados como positivos e negativos, onde os impactos positivos são relacionados a geração de empregos, causas sociais e doações, e os impactos negativos podem ser os acidentes de trabalho negligenciados, abusos de direitos humanos e assédio de empregados. A depender do contexto, a sócio-eficiência implica na minimização de impactos sociais negativos ou maximização dos positivos.

3) Suficiência: Caracteriza-se pela consciência de que existem recursos não substituíveis, de uso não linear e de causas irreversíveis, ou seja, a utilização dos recursos finitos no planeta deve ser respeitada, buscando assim uma padronização de uso que permita o não esgotamento deste material, evitando a extinção.

4) Eco-Eficácia: Caracteriza-se pela importante atenção de delimitar a eficiência, ou seja, o viés econômico do tripé pode induzir os gestores a elevar sua eficiência visando apenas o resultado financeiro, correndo assim o risco de alimentar em vez de reduzir a degradação ambiental. É papel da eficácia demonstrar o ponto de congruência entre ambiente e economia.

5) Sócio-Eficácia: Caracteriza-se pela dedicação econômica das organizações em fornecer seus produtos/serviços com excelência sem distinção de público, buscando sempre a positividade no impacto social. Ou seja, não fazer distinção entre países pobres e países ricos na distribuição de mercadorias, permitindo que a parte marginalizada da população seja alcançada.

6) Equidade: Caracteriza-se pelo equilíbrio entre a gestão do capital natural e a sociedade, ou seja, a criação de soluções para permitir que gerações futuras também tenham acesso aos recursos naturais hoje presentes na natureza. 
É de natureza econômica buscar o aumento dos índices financeiros das organizações, entretanto cabe aos agentes envolvidos no processo de desenvolvimento sustentável corporativo equilibrar valores políticos e étnicos aos resultados financeiros da organização, promovendo assim, a sustentabilidade corporativa.

\section{CONCLUSÃO}

A mensuração da sustentabilidade por meio de indicadores ainda não é um consenso e se encontra em fase de maturação e desenvolvimento científico, assim o presente trabalho teve o objetivo elencar alguns dos modelos atualmente aceitos.

Nesse sentido, a questão da sustentabilidade está se tornando cada vez mais importante para as empresas e o principal objetivo dos indicadores de desenvolvimento sustentável é fornecer informações aos tomadores de decisão sobre o nível geral de sustentabilidade de um sistema, bem como estabelecer um parâmetro que reflita o grau de sustentabilidade das empresas.

O desenvolvimento e aplicabilidade dos indicadores é muito importante, pois o mercado internacional tem se preocupado cada dia mais com a sustentabilidade do planeta

e ter índices positivos pode influenciar não somente na competitividade, como internamente com redução de custos, aumento na qualidade do produto ou serviço, melhoria da imagem do produto/serviço e da empresa, maior responsabilidade social.

Conclui-se, portanto, que o uso dos indicadores necessita de estudos mais profundos e também de individualização por atividades, pois ainda não existe um consenso universal de aplicabilidade de um método para todos os tipos de indpustria.

\section{REFERÊNCIA BIBLIOGRÁFICAS}

AKTAS, R.; KAYALIDERE, K.; KARĞIN, M. Corporate Sustainability Reporting and Analysis of Sustainability Reports in Turkey. International Journal of Economics and Finance-5 (3): 113-125, 2013.

ANDRADE, J. M.R.; CÂMARA, R.P.B. Mensuração da sustentabilidade empresarial: uma aplicação em hotéis localizados na via costeira da cidade de Natal/RN. Revista Ambiente Contábil - 4 (2): IIO - I31, 2012

AZAPAGIC, A.; PERDAN, S. Indicators of sustainable development for industry: a general framework. Process Safety and Environmental Protection, v.7, n.4, p.243-261, 2000. 
BARBIERI, J. C. Gestão ambiental empresarial: modelos e instrumentos. 4. ed. São Paulo: Saraiva, 2016.

BRUNDTLAND, G. H. Global change and our common future. Environment.1989;31(5):16.

COSTA, N.C.O.; SILVA, R.M.; CAMPOS, P.D.G.C.; SOUZA, T.L.. "A Engenharia de Produção e as novas tecnologias produtivas: indústria 4.0, manufatura aditiva e outras abordagens avançadas de produção" in XXXVII ENCONTRO NACIONAL DE ENGENHARIA DE PRODUCAO. Joinville, 2017. Io modelos que mensuram a sustentabilidade empresarial. Joinville, 2017.

DELAI, I.; TAKAHASHI, S. Uma proposta de modelo de referência para mensuração da sustentabilidade corporativa. RGSA - Revista de Gestão Social e Ambiental - 2 (I): 19-40, 2008.

DIAS, Reinaldo. Gestão ambiental: responsabilidade social e sustentabilidade. 3. ed. São Paulo: Atlas, 2019.

DYLLICK, T.; HOCKERTS, K. Beyond the business case for corporate sustainability. Business Strategy and the Environment, v. II, n. 2, p. 130-I4I, mar. 2002.

ELKINGTON, J., I994. Towards the suitable corporation: win-win-win business strategies for sustainable development. Calif. Manag. Rev. 36 (2), goeroo.

Finkbeiner, M., Schau, E. M., Lehmann, A., \& Traverso, M. (2010). Towards life cycle sustainability assessment. Sustainability, 2(10), 3309-3322.

GIMENEZ, C., Sierra, V., RODON, J., 2012. Sustainable operations: their impact on the triple bottom line. Int. J. Prod. Econ. I40 (I), I49 ei59.

Janeiro, L., \& Patel, M. K. (2015). Choosing sustainable technologies. Impli- cations of the underlying sustainability paradigm in the decision- making process. Journal of Cleaner Production, I05, 438-446.

KRONEMBERG, D. Desenvolvimento local sustentável: uma abordagem prática. Ed. Senac: São Paulo, 2or

Luke, T. W. (2005). Neither sustainable nor development: Reconsidering sustainability in development. Sustainable Development, ${ }_{13}(4), 228-238$.

MARCONI, M. A. Fundamentos de metodologia científica. 9 ed. São Paulo: Atlas, 202ı.

NOGUEIRA, E. P.; FARIA, A. C. Sustentabilidade nos principais bancos brasileiros: uma análise sob a ótica da global reporting initiative. Revista Universo Contábil, v. 8, p. II9-I39, 2012. 
SEARCY, C.; KARAPETROVIC, S.; MCCARTNEY, D. Designing sustainable development indicators: analysis for a case utility. Measuring Business Excelence - 9 (2): 33-41, 2005.

STIVAl, C. E.; SOTTORIVA, P. R. DA S. Sistema de Avaliação Ambiental para Projetos de Edificações Públicas (SAAPE): análise dos critérios de sustentabilidade de três projetos de edificações elaborados para a Universidade Federal do Paraná. Desenvolvimento e Meio Ambiente, v. 36, 30 abr. 2016.

SZÉKELY, F.; KNIRSCH, M. Responsible leadership and corporate social responsibility: metrics for sustainable performance. European Management Journal - 23 (6): 628-647, 2005 .

TACHIZAWA, Takeshy. Gestão ambiental e responsabilidade social corporativa: os paradigmas do novo contexto empresarial. 9. ed. São Paulo: Atlas, 2019.

TERRY, A., HILL, J. Sustainable Development: National Aspirations, Local Implementation (English Edition)

Van Marrewijk, M., \& Werre, M. (2003). Multiple levels of corporate sus[I]tainability. Journal of Business Ethics, 44(2), 107-119. 